\title{
Mulheres diagnosticadas com câncer de mama: impacto do crescimento pós-traumático
}

\author{
Maria Jeane Camargo* \\ Randolfo dos Santos Junior** \\ Loiane Letícia dos Santos*** \\ Bianca Vessecchi Talhaferro**** \\ Aline Monique Carniel***** \\ Ana Márcia Sanches de Almeida Vianna******
}

\begin{abstract}
Resumo
Câncer é possivelmente a doença mais temida na atualidade. Por seu caráter crônico, exige ajustamentos em múltiplos domínios na vida do paciente, com evidências indicando que fatores físicos, emocionais, cognitivos, interpessoais e comportamentais estão inter-relacionados e contribuem para o ajustamento de cada indivíduo. A perspectiva de superação de uma experiência desafiadora como o câncer pode resultar no desenvolvimento do que alguns autores denominam de crescimento pós-traumático. O objetivo consistiu em identificar os fatores relacionados ao crescimento pós-traumático a partir do diagnóstico e tratamento de câncer de mama. Participaram deste estudo trinta mulheres diagnosticadas com câncer de mama em tratamento no Hospital de Base. Para o processo de coleta de dados foram utilizados, questionário sociodemográfico, Escala de Ansiedade e Depressão Hospitalar, Escala de esperança de Herth, Escala de Apoio Social, Escala de Ajustamento Mental para o câncer e Inventário de Crescimento Pós-Traumático. Como resultado verificou-se que variáveis como idade, possuir um companheiro, maior tempo de diagnóstico, esperança, menores indicadores de depressão, maior nível de escolaridade e religião são fatores que colaboram para o desenvolvimento do crescimento pós-traumático. Estes resultados apontam para a importância da identificação destas variáveis no perfil psicossocial de pacientes com câncer, e principalmente para a necessidade de elaborar intervenções que possam promover a manutenção e o desenvolvimento de fatores de proteção em face aos estressores do tratamento.
\end{abstract}

Palavras-chave: Neoplasia Mamária, Crescimento Pós-traumático; Saúde Mental

\section{Women diagnosed with breast cancer: Impact of post-traumatic growth}

\begin{abstract}
Cancer is possibly the most feared disease today. Due to its chronic nature, it requires adjustments in multiple domains in the patient's life, with evidence indicating that physical, emotional, cognitive, interpersonal and behavioral factors are interrelated and contribute to the adjustment of each individual. The prospect of overcoming a challenging experience like cancer can result in the development of what some authors call post-traumatic growth. The objective was to identify factors related to post-traumatic growth from the diagnosis and treatment of breast cancer. Thirty women diagnosed with breast cancer undergoing treatment at the Base Hospital participated in this study. For the data collection process, a sociodemographic questionnaire, Hospital Anxiety and Depression Scale, Herth's Hope Scale, Social Support Scale, Mental Adjustment Scale for cancer and Post-Traumatic Growth Inventory were used. As a result, it was found that variables such as age, having a partner, longer diagnosis time, hope,
\end{abstract}

* Psicóloga pela UNORP e aprimoranda pela FAMERP.

** Psicólogo pela UFU, Doutor pela FAMERP e Prof. Adjunto na FAMERP

*** Psicóloga pela FQM, Mestre pela FAMERP e Doutoranda na UNICAMP.

**** Psicóloga pela União das Faculdades dos Grandes Lagos e Mestranda pela FAMERP

****** Psicóloga pelo CUNP e Especialista em Oncologia pelo PRMA ao Câncer da FAMERP em parceria com o Hospital de Base de São José do Rio Preto/SP.

****** Psicóloga pela Fundação FAMERP e Mestre pela FAMERP. 
lower indicators of depression, higher level of education and religion are factors that contribute to the development of post-traumatic growth. These results point to the importance of identifying these variables in the psychosocial profile of cancer patients, and mainly to the need to develop interventions that can promote the maintenance and development of protective factors in the face of treatment stressors.

Key-words: Breast Neoplasms; Post-Traumatic Growth; Mental Health.

\section{Introdução}

Segundo dados do Instituto Nacional do Câncer (INCA, 2019) a neoplasia da mama é o tipo mais comum entre as mulheres no Brasil e no mundo, seguido do de pele não melanoma, correspondendo cerca de $25 \%$ dos casos novos a cada ano, sendo que em 2016, foram registrados no Brasil, 16.069 óbitos por câncer de mama em mulheres e a estimativa para o ano de 2020 é de 66.280 novos casos (INCA, 2020).

Câncer é possivelmente a doença mais temida na atualidade, o de mama especificamente origina-se pela multiplicação desordenada das células no local, gerando células anormais que se multiplicam, formando um tumor. A detecção precoce do diagnóstico possibilita maiores chances do sucesso do tratamento e diminui intervenções agressivas (INCA, 2019).

Dentre os fatores de risco para o câncer de mama o envelhecimento e a vida reprodutiva da mulher, como menarca precoce, não ter tido filhos, e menopausa tardia possuem grande influência para o surgimento da doença. No entanto, os fatores de proteção para reduzir sua ocorrência estão intimamente relacionados com evitar o consumo de bebida alcoólica, manter a alimentação saudável e a prática regular de atividade física (INCA, 2019).

O diagnóstico de câncer para muitas pessoas significa uma aproximação direta com a perspectiva de morte, mutilação, dor e incurabilidade (Silva \& Melo-Santos, 2008; Reich, Gaudron \& Penel, 2009), uma vez que traz para o paciente a perda do corpo saudável, da sensação de invulnerabilidade e do domínio sobre a própria vida (Rossi, \& Santos, 2003). Um estudo realizado com mulheres após receber o diagnóstico de câncer de mama demonstrou que sentimentos como o medo da morte, tristeza, desespero e preocupação com a família estão presentes nesse momento (Barros, Conde, Lemos, Kunz, \& Ferreira, 2018).

Por seu caráter crônico, exige ajustamentos em múltiplos domínios na vida do paciente, com evidências indicando que fatores físicos, emocionais, cognitivos, interpessoais e comportamentais estão inter-relacionados e contribuem para o ajustamento final observado em cada indivíduo (Larsen \& Hummel, 2008; Santos, Simone, Pinhabel, Galhardo, \& Carniel, 2019).
Frente às mudanças ocasionadas em decorrência do diagnóstico, Silva (2019) discute a respeito das alterações emocionais vivenciadas pelo paciente oncológico como sintomas depressivos, ansiosos, isolamento, medos, angústias e estresse. Esse último considerado como uma relação entre a pessoa e o ambiente, avaliado por ela como exigente ou excedente aos seus recursos pessoais de enfrentamento e ameaçador ao seu bem-estar (Andreotti, Root, McEwen, \& Compas, 2015).

Dessa forma, a partir da interpretação que as pessoas fazem sobre um evento, ocorre o que alguns autores denominam de avaliação cognitiva, sendo esse um processo pelo qual a pessoa categoriza uma situação e seus aspectos em relação ao seu potencial ameaçador (Hopman \& Rijken, 2015).

Existem dois tipos de avaliação cognitiva, a avaliação primária, que categoriza uma situação como irrelevante, quando não é percebida como prejudicial, benigna quando é avaliada como positiva, proporcionando bem-estar ou estressante, quando é tida como fatigante, passível de causar danos ou perdas. O segundo tipo de avaliação, a secundária está associada ao que poderá ser feito para lidar com a situação em particular. Ou seja, os esforços necessários para superar, controlar ou evitar repercussões e danos posteriores (Folkman, Lazarus, \& DeLongis 1986; Lawrenz \& Castro, 2011; Veras, Veras Jr. \& Carvalho, 2015).

Estratégias de coping ou enfrentamento são os esforços cognitivos e comportamentais do indivíduo para administrar e gerenciar as demandas internas e externas da relação (pessoa-ambiente), avaliadas como superiores aos recursos individuais, isto é, o manejo de situações potencialmente danosas ou desafiadoras (Veras, Veras, Jr. \& Carvalho, 2015; Castro, Lawrenz, Romeiro, Lima \& Haas, 2016).

Dell'Aglio (2000) conclui que quando o coping é direcionado para uma fonte externa, pode abarcar estratégias de negociação ou solicitação de auxílio de outras pessoas, mas quando é direcionado para uma fonte interna, normalmente envolve uma reestruturação cognitiva, para avaliar a situação de forma mais adaptativa.

Após a avaliação inicial do acontecimento como estressante é iniciado o processo de enfrentamento em que o paciente irá utilizar determinadas estratégias, podendo ser tanto focadas na emoção quanto no problema, 
tendo como objetivo minimizar o impacto do evento em sua vida (Tavares \& Trad, 2009; Silva, Zandonade \& Amorim, 2017).

Diante da premissa de que o ser humano adoece em sua totalidade e considerando o impacto do diagnóstico de câncer em seu psiquismo, o Ministério da Saúde (1998) no Serviço de Suporte, estabelece a obrigatoriedade do profissional da psicologia nos centros de atendimento em oncologia - Portaria, 3.535, uma vez que o mesmo irá atuar por meio do apoio psicoterapêutico (Scannavino, Sorato, Lima, Franco, Martins, Morais, Bueno, Rezende \& Valério, 2013), auxílio ao paciente no ajustamento psicossocial diante da doença (Cardoso, Alberton, Fernandes \& Castro, 2019), além de desmistificar os medos e fantasias decorrentes do diagnóstico (Beck, 2017).

Nesse sentido, nas últimas décadas há uma série de estudos longitudinais que retratam as transformações vivenciadas pelo ser humano após determinado evento traumático, como: residentes de um centro de acolhimento em Lisboa (Fonseca, 2011); jovens-adultos que experienciaram algum evento potencialmente traumático (Santos, 2017); valores humanos (Medeiros, Couto, Fonseca, Brito, \& Castro, 2016), perdão no âmbito do divórcio (Couto, 2017); traumas de mães e seus recém-nascidos (Diab, Isosävi, Qouta, Kuittinen, \& Punamäki, 2018).

Todos esses estudos demonstram que em face da perspectiva de superação de uma experiência desafiadora, muitas pessoas relatam uma maior valorização e satisfação com a vida e um maior senso de propósito permeado por uma perspectiva de ajustamento positivo e as experiências ocorridas foram denominadas por Tedeschi e Calhoun (1996) de crescimento pós-traumático - CPT.

Tendo em vista que o crescimento pós-traumático é considerado uma resposta adaptativa a um estressor grave e que este processo implica em uma capacidade do indivíduo de elaborar uma reestruturação cognitiva por vezes profunda de crenças, expectativas e propósitos de vida, pode-se considerar a importância de aspectos pessoais e ambientais que podem favorecer essa tarefa de ressignificação. Pesquisas apontam que o crescimento pós-traumático pode ser favorecido por características pessoais como: flexibilidade emocional, saúde mental e presença de sentido (Schroevers, Helgeson, Sanderman \& Ranchor, 2010; Casellas-Grau, Ochoa \& Ruini (2017); enfrentamento religioso positivo e otimismo (Prati \& Pietrantoni, 2009); percepção de apoio emocional significativo (Zeligman, Varney, Grad, \& Huffstead, 2018) e do suporte social como mediador para melhor autoestima e maiores indicadores de esperança (Zhou, Wu, Zhen, 2018).
As questões explanadas acima apontam para a importância da identificação destas variáveis no perfil psicossocial de pacientes com câncer, dessa forma o objetivo da presente pesquisa consistiu em identificar os fatores relacionados ao crescimento pós-traumático a partir do diagnóstico e tratamento de câncer de mama.

\section{Casuística e método}

\section{Participantes}

Participaram do presente estudo trinta mulheres, que correspondiam aos seguintes critérios: diagnóstico de câncer de mama em tratamento no Hospital de Base (HB), Instituto do Câncer (ICA) e Unidade de Quimioterapia, adultos (idade superior a 18 anos) e em tratamento há pelo menos 3 (três) meses - período mínimo para ajustamento psicossocial. Foram excluídos participantes com dificuldades de audição e ou/ fala, ou com déficit cognitivo apontado em prontuário. A coleta ocorreu entre os meses de outubro de 2019 a janeiro de 2020, o tempo que as participantes utilizaram para responder os instrumentos foi em média 35 minutos.

Foram consultados os prontuários de 50 pacientes sendo que trinta e sete mulheres preenchiam os critérios de inclusão mencionados acima, três não aceitaram participar do estudo, quatro daqueles que estavam na sala de espera do Instituto do Câncer (ICA) aceitaram, mas não finalizaram por desistência ou por terem sido chamados para a consulta médica, por fim trinta aceitaram e concluíram a pesquisa.

\section{Fluxograma da amostra}

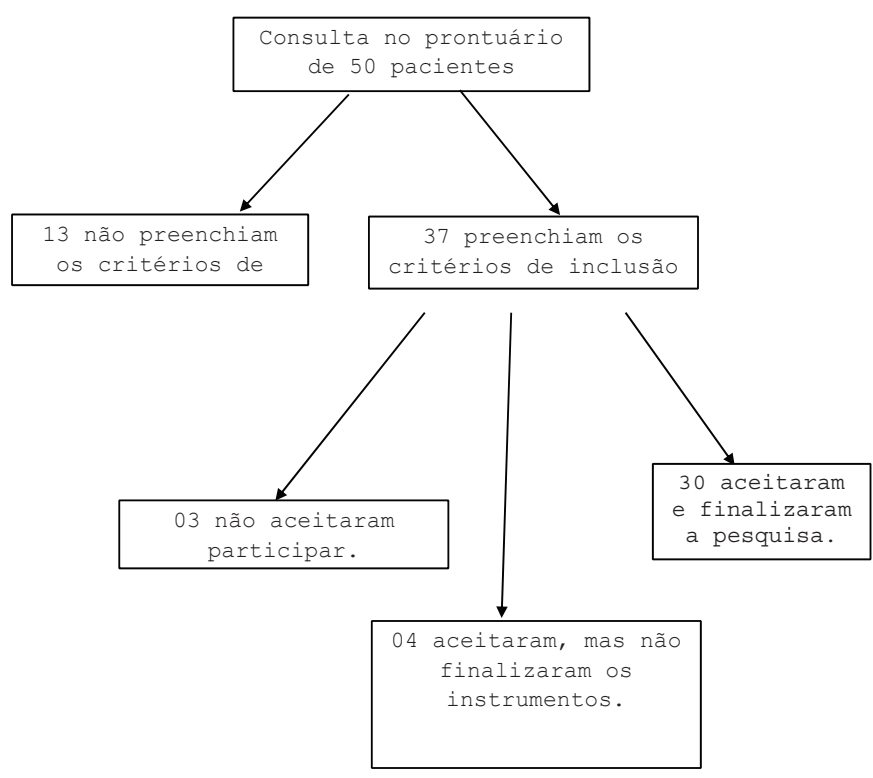

Mudanças - Psicologia da Saúde, 28 (1) 17-26, Jan.-Jun., 2020 


\section{Materiais}

Para o processo de coleta de dados, as participantes responderam aos seguintes instrumentos:

Questionário inicial de dados sociodemográficos: Instrumento de autoria dos pesquisadores foi construído com o objetivo de caracterizar os participantes quanto aos aspectos demográficos e clínicos, que investiga: na primeira parte - (através de uma pesquisa ao prontuário do paciente): diagnóstico; tempo de diagnóstico, presença de metástase, na segunda parte, (indagando ao próprio paciente): idade, sexo, endereço, escolaridade; estado civil; quantidade de filhos; raça; atividade profissional; religião. (Apêndice B).

Escala de Ansiedade e Depressão Hospitalar - HAD (Escala para avaliar ansiedade e depressão em adultos): Suas principais características são: sintomas vegetativos que podem aparecer em doenças físicas foram evitados; os conceitos de depressão e ansiedade encontram-se separados, o conceito de depressão encontra-se centrado na noção de anedonia; inclina-se a detectar graus leves de transtornos afetivos em ambientes não psiquiátricos; é curta, podendo ser rapidamente aplicada e preenchida. Ao paciente solicita-se e é recomendado que responda baseando-se em como se sentiu durante a última semana. Sendo 14 questões de múltipla escolha e duas sub-escalas (ansiedade e depressão) com sete itens cada, (ponto de corte 8). Serão adotados os pontos de corte apontados por Zigmond e Snait (1983), recomendados para ambas as situações: de 0-7 pontos: improvável, 8-11 pontos: possível (questionável ou duvidoso) e 12-21: provável. As análises serão feitas a partir das normas propostas de acordo com a validação elaborada por Botega, Bio, Zomignani, Garcia Jr., \& Pereira, (1995).

Escala de esperança de Herth: A escala originou-se do instrumento americano Hert Hope Index e possibilitou a existência de um instrumento específico para mensuração da esperança, antes inexistente no Brasil, chamada de Escala de Esperança de Herth (EEH). É uma escala de autorrelato de origem americana, que quantifica a esperança de vida. Esta escala possui 12 afirmativas e a graduação dos itens segue a escala do tipo Likert de quatro pontos, variando de "concordo completamente" a "discordo completa mente" onde 1 indica "discordo completamente" e 4 corresponde a "concordo completamente". Os itens 3 e 6 apresentam escores invertidos. $\mathrm{O}$ escore total varia de 12 a 48 e quanto maior o escore, mais alto o nível de esperança de vida. É uma escala considerada breve e de fácil compreensão e demonstrou prioridades psicométricas adequadas (Sartore, \& Grossi, 2008).

Escala de Apoio Social: Composto por 19 itens compreendendo cinco dimensões funcionais de apoio social: material, afetiva, emocional, interação social positiva e informação (Griep, Chor, Faerstein, Werneck, \& Lopes, 2005). Visa avaliar em que medida a pessoa conta com o apoio de outras para enfrentar situações estressantes (Zanini, Primi \& Peixoto, 2018).

Escala de Ajustamento Mental para o câncer (MINI-MAC): Trata-se de um instrumento constituído por 2 (dois) fato- res: Fator 1 - Preocupação Ansiógena: apresenta 8 (oito) afirmações como, por exemplo, "Estou preocupada com minha doença" e "Sinto-me agoniada com minha doença"; Fator 2 - Desamparo/Desesperança: apresenta 5 (cinco) afirmações, entre elas, "Sinto que esta doença está ale, das minhas forças" e "Não consigo lutar contra minha doença". Nos dois fatores que constituem a escala, as respostas para as afirmações são apresentadas desde "Nunca" até "Sempre" em uma escala do tipo Likert de 4 pontos, onde conforme maiores os valores apresentados piores as condições de Preocupação Ansiógena e Desamparo/ Desesperança (Gandini, Martins e Pedrosa, 2008).

Inventário de Crescimento Pós-Traumático: É composto por 18 itens organizados em uma estrutura multifatorial composta por cinco fatores: relação com os outros, novas possibilidades, mudança pessoal, e apreciação da vida. Ele é respondido a partir de uma escola Likert de 6 pontos $(0 \mathrm{a}$ 5), tendo como referência o grau de mudanças apresentadas após uma crise específica, sendo que o valor total (somatório de todos os itens) corresponde ao índice de crescimento pós-traumático (Tedeschi \& Calhoum, 1996).

\section{Procedimentos}

Inicialmente, os pesquisadores elaboraram o projeto de pesquisa e o submeteram ao Comitê de Ética em Pesquisa com Seres Humanos (CEP/FAMERP). Após aprovação (parecer $n^{\circ} 3494109$ ) foi necessário entrar no prontuário eletrônico das pacientes para verificar se as mesmas entravam nos critérios para participarem da pesquisa.

A partir dessa busca, as pacientes que estavam em tratamento oncológico no Instituto do Câncer (ICA), e Hospital de Base de São José do Rio Preto (HB), foram convidadas durante consulta no Instituto do Câncer, hospitalização e nas sessões de quimioterapia a participarem do estudo.

No início dos atendimentos foram realizados os esclarecimentos necessários sobre a utilização acadêmica e científica dos dados e foi apresentado e realizado leitura do termo de Consentimento Livre e Esclarecido (Modelo em acordo com a Resolução $n^{\circ}$ 466/12 - Conselho Nacional de Saúde), contendo dados de identificação do pesquisador e dos participantes, objetivos e procedimentos da pesquisa (Apêndice A). Os participantes que concordaram assinaram o Termo de Consentimento Livre e Esclarecido

Análise de dados

Para análise dos dados foram utilizados estatística descritiva, apresentação dos dados clínicos e sociodemográficos e o Teste de Spearman para a análise de correlação entre as variáveis com nível de significância de $\mathrm{p}<0,05$.

\section{Resultados}

A amostra foi composta de 30 mulheres, sendo casadas (70\%), idade média de 50 anos e com o tempo de convívio com a doença variando entre menos de 12 meses (17\%) e mais de 49 meses (36\%).

No que diz respeito à ocupação 70\% afirmaram exercer alguma atividade profissional e duas encontravam-se 


\section{MULHERES DIAGNOSTICADAS COM CÂNCER DE MAMA: IMPACTO DO CRESCIMENTO PÓS-TRAUMÁTICO}

aposentadas (7\%). A religião predominante foi a evangélica $(53 \%)$, seguida pela católica $(32 \%)$.

Quanto à escolaridade, observou-se que grande maioria das mulheres entrevistadas possuía o $1^{\circ}$ grau incompleto $(33 \%), 2^{\circ}$ grau completo $(30 \%)$ e $10 \%$ relataram terem finalizado o ensino superior.
Em relação à presença de transtorno mental 23 (77\%) relataram nunca terem sido diagnosticadas com algum transtorno mental, $5(17 \%)$ das pacientes afirmaram já terem sido diagnosticadas com depressão em algum momento da vida e $2(6 \%)$ já foram diagnosticadas com algum tipo de ansiedade (Tabela 1).

Tabela 1: Caracterização da amostra de pacientes oncológicos em acompanhamento pelo Serviço de Mastologia de um Hospital Geral, do Interior de São Paulo, 2019.

\begin{tabular}{|c|c|c|c|c|c|}
\hline Variáveis & $\mathbf{N}$ & $\%$ & Variáveis & $\mathbf{N}$ & $\%$ \\
\hline Local da coleta & & & Frequência à igreja & & \\
\hline Instituto do Câncer (ICA) & 25 & 85 & Nunca & 4 & 14 \\
\hline Enfermaria - Hospital de Base & 3 & 10 & Diariamente & 1 & 3 \\
\hline Quimioterapia & 2 & 5 & Uma vez por semana & 9 & 30 \\
\hline Tipos de câncer (Neoplasia primária) & & & Duas ou mais vezes por semana & 9 & 30 \\
\hline Câncer da mama esquerda & 15 & 49 & Menos que uma vez por mês & 3 & 10 \\
\hline Câncer da mama direita & 14 & 46 & Uma/duas vez por mês & 4 & 13 \\
\hline Carcinoma lobular da mama & 1 & 5 & Reside com & & \\
\hline Metástase & & & Sozinha & 2 & 7 \\
\hline $\operatorname{Sim}$ & 1 & 3 & Com o marido/companheiro & 7 & 23 \\
\hline Não & 29 & 97 & Com marido e filhos & 8 & 27 \\
\hline Idade & & & Com filhos & 6 & 20 \\
\hline Entre 30 e 40 anos & 5 & 17 & Outros & 7 & 23 \\
\hline Entre 40 e 50 anos & 8 & 27 & Ocupação & & \\
\hline Entre 50 e 60 anos & 10 & 33 & Autônoma & 7 & 23 \\
\hline A partir de 60 anos & 7 & 23 & Auxiliar de limpeza & 4 & 13 \\
\hline Escolaridade & & & Do lar & 7 & 23 \\
\hline $1^{\circ}$ grau incompleto & 10 & 33 & Funcionária pública & 2 & 7 \\
\hline $1^{\circ}$ grau completo & 2 & 7 & Outra & 10 & 34 \\
\hline $2^{\circ}$ grau incompleto & 3 & 10 & Cidade de Origem & & \\
\hline $2^{\circ}$ grau completo & 9 & 30 & Votuporanga & 5 & 17 \\
\hline Universitário incompleto & 3 & 10 & São José do Rio Preto & 7 & 23 \\
\hline Universitário completo & 3 & 10 & Guapiaçu & 2 & 7 \\
\hline Estado civil & & & Outras & 16 & 53 \\
\hline Casada & 17 & 70 & Tempo do diagnóstico & & \\
\hline Solteira & 3 & 13 & Menos de 12 meses & 5 & 17 \\
\hline Divorciada & 3 & 12 & Até 24 meses & 6 & 20 \\
\hline Possui companheiro & 7 & 5 & Até 48 meses & 8 & 27 \\
\hline Cor/raça & & & Mais de 49 meses & 11 & 36 \\
\hline Branca & 17 & 56 & Transtorno psicológico & & \\
\hline Preta & 1 & 3 & Nenhum & 23 & 77 \\
\hline Parda & 11 & 37 & Depressão & 5 & 17 \\
\hline Amarela & 1 & 4 & Ansiedade & 2 & 6 \\
\hline Religião & & & Filhos & & \\
\hline Católica & 9 & 32 & Nenhum & 2 & 7 \\
\hline Evangélica & 15 & 53 & $\mathrm{Um}$ & 8 & 27 \\
\hline Espírita & 2 & 7 & Dois & 14 & 46 \\
\hline Budista & 1 & 4 & Três & 5 & 17 \\
\hline Outra & 1 & 4 & Mais de três & 1 & 3 \\
\hline
\end{tabular}


Quando examinado os resultados dos instrumentos foi possível perceber que na Escala Hospitalar de Ansiedade e Depressão-HAD, o escore de ansiedade $(6,6)$ demonstrou maior prevalência quando comparado com o de depressão $(5,2)$. Para verificar a presença de esperança entre as participantes aplicou-se a Escala de Esperança e obteve-se como resultado o escore 38,4. Ademais observou-se que na Escala de Apoio Social o subtipo afetivo $(465,4)$ apresentou maior prevalência entre as participantes. Por meio da Escala de Ajustamento Mental para o Câncer constatou-se que a preocupação foi a emoção mais vivenciada pelas participantes $(3,8)$ (Tabela 2).

Tabela 2. Resultados obtidos através da Escala Hospitalar de Ansiedade e Depressão, Escala de Esperança, Escala de Ajustamento Mental par o Câncer e Inventário de Crescimento Pós-traumático aplicados em mulheres com câncer de mama de um Hospital Geral do interior de São Paulo.

\begin{tabular}{l|l}
\hline Instrumentos & \multicolumn{1}{|c}{ Escore } \\
\hline HAD - Ansiedade & 6,6 \\
\hline HAD - Depressão & 5,2 \\
\hline HAD - Total & 11,9 \\
\hline Escala de Esperança (EEH) & 38,4 \\
\hline Escala de apoio Social - Parentes & 2,9 \\
\hline Escala de apoio Social - Amigos & 2,8 \\
\hline Escala de apoio Social - Material & 423,33 \\
\hline Escala de apoio Social - Afetivo & 465,4 \\
\hline Escala de apoio Social - Emocional & 406,6 \\
\hline Escala de apoio Social - Informação & 383,3 \\
\hline Escala de apoio Social - Interação & 410,8 \\
\hline Escala de apoio Social - Total & 2067,6 \\
\hline Escala de Ajustamento Mental para o Câncer - Preocupação & 3,8 \\
\hline Escala de Ajustamento Mental para o Câncer - Desamparo & 0,9 \\
\hline Escala de Ajustamento Mental Para o Câncer - Total & 4,7 \\
\hline Inventário de Crescimento Pós-Traumático & 70,6 \\
\hline
\end{tabular}

Utilizou-se o Teste de Spearman para a análise de correlação entre o crescimento pós-traumático e as variáveis, sendo que o tempo de diagnóstico, escolaridade, depressão e esperança obtiveram correlação significativa para a presença de crescimento pós-traumático dentre as participantes do presente estudo (Tabela 3).

Tabela 3. Correlações entre a escala de crescimento pós-traumático e as variáveis tempo do diagnóstico, escolaridade, ansiedade, depressão, apoio social, idade, esperança e preocupação de mulheres com câncer de mama de um Hospital Geral do interior de São Paulo.

\begin{tabular}{c|l|l}
\hline Variáveis & $\mathbf{r}$ & $\mathbf{p}$ \\
\hline Tempo do diagnóstico & 0,3844 & $0,036^{*}$ \\
\hline Escolaridade & 0,3671 & $0,046^{*}$ \\
\hline Ansiedade & 0,1638 & 0,387 \\
\hline Depressão & $-0,4009$ & $0,028^{*}$ \\
\hline Apoio Social & 0,2689 & 0,151 \\
\hline Idade & $-0,113$ & 0,550 \\
\hline Esperança & 0,4457 & $0,014^{*}$ \\
\hline Preocupação & $-0,0363$ & 0,849 \\
\hline
\end{tabular}

*Teste de Spearman nível de significância de $\mathrm{p}<0,05$. 


\section{Discussão}

O tempo de diagnóstico do câncer de mama neste estudo foi relacionado com maior presença de crescimento pós-traumático. Em um estudo longitudinal foi observado que com o passar do tempo estratégias de enfrentamento positivas e CPT demonstraram estar presentes principalmente após os seis primeiros meses após o diagnóstico, no entanto sete anos após a descoberta, não havia mais indicadores de crescimento pós-traumático (Raz-Hamama, Pat-Horenczyk, Roziner, Perry \& Stemmer, 2019).

Em pesquisa realizada com pacientes também com câncer de mama, Wang, Liu, Wang, Chen e Li, (2014) obtiveram como resultado índices mais altos de CPT em pacientes que possuíam maior nível de escolaridade. Tal fato pode ser justificado, por esse perfil de pessoas enxergarem determinado evento traumático de forma mais ampla e exibirem um estilo explicativo mais otimista diante de tal evento (Ho, Chan, Yau, \& Yeung, 2010).

O diagnóstico de uma neoplasia, especificamente a da mama acarreta uma série de mudanças vivenciadas pela mulher ao longo do tempo, como, por exemplo, o impacto na sua imagem corporal. No entanto o diagnóstico não traz demandas apenas sobre aspectos físicos, mas também sociais e psíquicos. Dito isso, em um estudo observacional, a respeito da ocorrência de depressão e ansiedade em mulheres diagnosticadas com câncer de mama, foi verificado que a prevalência de depressão, ansiedade ou ambas no ano após o diagnóstico é cerca de duas vezes maior a da população feminina geral (Burguess, Cornelius, Love, Graham, Richards, \& Ramirez, 2005).

Diante dos efeitos psicológicos causados por um evento estressor como o câncer e a maneira como cada sujeito pode responder a ele, observou-se que dentre os participantes desta pesquisa houve uma correlação negativa entre a sintomatologia de depressão e crescimento pós-traumático. Ou seja, quanto maior o nível percebido de depressão do sujeito, menor os indicadores de CPT. Tal resultado é consistente com estudo realizado na Universidade do Alabama (EUA) onde foi apresentado relação negativa entre depressão e crescimento pós-traumático (LaRocca, Scogin, Hilgeman, Smith, \& Chaplin, 2018).

Embora a ansiedade não tenha apresentado relevância nesta pesquisa para indicar o crescimento pós-traumático, outro estudo recente (Chen, Chen, Hsiao, Weng, Hsu, Wung, Su \& Chen, 2019) demonstrou que sintomas de ansiedade associados com variáveis como maior nível de escolaridade e apoio familiar predizem o CPT. Segundo esses autores, sintomas ansiosos após o câncer podem permitir que os pacientes tenham outras oportunidades durante o crescimento pós-traumático.

De forma a complementar a esse achado, Groarke, Curtis, Groarke, Hogan, Gibbons \& Kerin (2017) mostraram que a ansiedade como consequência de um diagnóstico em estágio inicial de câncer pode apresentar relação positiva com o CPT. No entanto, outros estudos consultados apontam para a associação negativa entre depressão, ansiedade e CPT (Val \& Linley, 2006; Salsman, Segerstrom, Brechting, Carlson, \& Andrykowski, 2008).

Como já abordado anteriormente, sabe-se que os benefícios percebidos, aprendizagens e aspectos positivos decorrentes do trauma colaboram para o desenvolvimento do crescimento pós-traumático (Santos, Simone, Pinhabel, Galhardo, \& Carniel, 2019). No entanto, depois de um período em que se dá a descoberta do diagnóstico e das abordagens terapêuticas, as estratégias de enfrentamento para o crescimento pós-traumático não se mostram mais tão eficazes como no início (Raz-Hamama, Pat-Horenczyk, Roziner, Perry \& Stemmer, 2019).

Por meio da tabela 3 é possível observar que no presente estudo as mulheres diagnosticadas com câncer de mama não apresentaram preditores significativos de apoio social relacionado ao CPT. Tal dado diverge de alguns autores que apresentam uma correlação positiva entre o crescimento pós-traumático e o apoio social como uma estratégia que colabora para o paciente lidar com os desafios e dificuldades do diagnóstico de câncer (Hasson-Ohayon, Tuval-Mashiach, Goldzweig, Levi, Pizem \& Kaufman, 2016).

No entanto, Prati e Pietrantoni (2009) verificaram através de uma revisão de 103 estudos que o apoio social está apenas moderadamente relacionado ao crescimento pós-traumático. Em síntese, os dados da presente pesquisa somam-se aos da literatura existente no que diz respeito à ambiguidade de resultados que prediz a relação positiva entre o apoio social e CPT.

As participantes dessa pesquisa podem ser caracterizadas como estando na meia-idade (45 a 65 anos). No estudo feito por Kroenke, Rosner, Chen, Kawachi, Colditz \& Holmes (2004), observou-se que mulheres mais jovens demonstraram um desempenho físico e psicossocial pior. Ou seja, maior sofrimento emocional e reajuste psicológico após o diagnóstico de câncer de mama em comparação com as mulheres mais velhas, em razão disso supõe-se o impacto do câncer difere de acordo com o estágio da vida em que a paciente se encontra.

Quando correlacionado com o crescimento pós-traumático verificou-se por meio desse estudo que a 
menor idade é uma variável que prediz o CPT. Autores como Boyle, Stanton, Ganz e Bower (2017) apontam que tal fato pode ser observado em pessoas mais jovens por meio do humor positivo, postura esperançosa e uma perspectiva voltada para o futuro. Ademais, Tedeschi \& Calhoun (2004) partem da premissa de que pessoas mais jovens podem relatar maior crescimento, pois tendem a estar mais abertas a aprenderem com as mudanças decorrentes do trauma em comparação com as mais velhas.

Apesar dos resultados desse estudo no que se refere à religião não terem demonstrado significância, a religião evangélica se mostrou predominante entre a amostra, dado que vai ao encontro da pesquisa realizada por Choumanova, Wanat, Barret e Koopman (2006) em que as mulheres descreviam a religião como o principal recurso para lidar com o diagnóstico de câncer. Por essa razão entende-se que aspectos religiosos também podem afetar a maneira como cada indivíduo enxerga o trauma (Tobin, Allem, Slaughter, Unger, Hamilton, \& Millam, 2017).

No tocante a variável esperança as descobertas desta pesquisa estão alinhadas com outro estudo recente (Todorov, Sherman e Kilby, 2019), que identificou a autocompaixão e esperança como fatores de proteção ao sofrimento psíquico. Também, o estudo de Baglama e Atak (2015) que evidenciou a correlação entre níveis mais altos de esperança e crescimento pós-traumático em pacientes diagnosticadas com câncer de mama. $\mathrm{O}$ mesmo resultado foi observado no presente estudo.

Durante a coleta de dados dessa pesquisa alguns pacientes relataram outros eventos traumáticos, como a perda de familiares, filhos (2), sobrinho (1)e um dos pais (2). A esse respeito, Tedeschi \& Calhoum (2004) discorrem que quanto mais traumático e avassalador for o evento maior e mais rápido o $\mathrm{CP} T$ se desenvolverá. No entanto quando indivíduos já passaram por um acontecimento traumático anterior, o impacto desse evento para que ocorra o CPT pode ser reduzido, visto que não é o primeiro evento traumático vivenciado, o que pode ajudar a compreender o motivo dessas mulheres não verem o câncer de mama como algo tão traumático comparado com as que não experienciaram outro evento traumático (Raz-Hamama, Pat-Horenczyk, Roziner, Perry \& Stemmer, 2019)

Ao interpretar os dados desse estudo algumas limitações devem ser discutidas, como o fato de as participantes terem vivenciado apenas um tipo de evento traumático, o câncer de mama. Outro aspecto foi o tamanho reduzido da amostra que impossibilitou resultados mais significativos, somando-se ao tempo limitado para a coleta de dados.

Ademais, o lugar da coleta de dados pode não ter sido o mais adequado, visto que a maioria das pacientes foram entrevistas na sala de espera de um ambulatório especializado, podendo assim terem ficado sujeitas há alguns distratores como ruídos e conversas paralelas, o que pode ter dificultado a concentração no momento de responder aos instrumentos.

\section{Conclusão}

O diagnóstico de câncer é um evento que provoca uma série de emoções que acompanha o paciente no decorrer do tratamento. Quando se trata de uma neoplasia mamária percebe-se que o impacto é sentido através dos estigmas sociais, mudanças na autoimagem, e nos relacionamentos. Portanto, para lidar com as consequências do adoecimento existem estratégias que cada pessoa irá utilizar de acordo com seu estilo de enfrentamento.

Em síntese, o crescimento pós-traumático surge como resposta a esses eventos, no entanto é um processo complexo que envolve características emocionais e cognitivas, sendo que através desse estudo observou-se que as participantes apresentaram indicadores significativos de CPT, sendo que variáveis como esperança, menor idade e nível de escolaridade mostraram-se fundamentais para sua presença.

Em suma as participantes apresentaram prováveis indicadores de ansiedade e depressão. Por conseguinte, achados a respeito da correlação entre depressão e CPT foram consistentes com outros estudos, porém quando diz respeito a ansiedade há uma relação ambígua sobre até que ponto essa é uma variável positiva para o crescimento pós-traumático.

Portanto é esperado que o presente trabalho sirva de incentivo para que novas pesquisas sejam realizadas na busca compreender melhor as variáveis que influenciam no crescimento pós-traumático não só de pessoas diagnosticadas com câncer, mas também daquelas que vivenciaram outro tipo de evento traumático, uma vez que como já foi discorrido, o crescimento pós-traumático não se limita ao diagnóstico de uma neoplasia maligna.

Além disso, estes resultados apontam para a importância da identificação destas variáveis no perfil psicossocial de pacientes com câncer, e principalmente para a necessidade de elaborar intervenções que possam promover a manutenção e o desenvolvimento de fatores de proteção em face dos estressores do tratamento, reforçando sempre a capacidade do ser humano de transcender mesmo em 
momentos de crises. Apesar das limitações explanadas, pretende-se que o presente estudo contribua para o corpo de literatura sobre o crescimento pós-traumático diante do diagnóstico do câncer de mama.

\section{Referências}

Andreotti, C., Root, C. J., Ahles, A. T., McEwen, S. B., \& Compas, E. B. (2015). Cancer, coping, and cognition: a model for the role of stress reactivity in cancer-related cognitive decline. Psycooncology. 24 (6), 617-623. Retrieved from https://pubmed.ncbi.nlm.nih.gov/25286084/ doi: 10.1002/pon.3683

Baglama, B. \& Atak, I. E. (2015). Posttraumatic Growth and Related Factors Among Postoperative Breast Cancer Patients. Procedia - Social and Behavioral Sciences. 190 (21), 448-454. Retrieved from https://www.sciencedirect.com/ science/article/pii/S1877042815032668 doi: 10.1016/j.sbspro.2015.05.024

Barros, A. E. S., Conde, C. R. C., Lemos, T. M. R., Kunz, J. A., Ferreira, M. L. S M. (2018). Sentimentos vivenciados por mulheres ao receberem o diagnóstico de câncer de mama. Revista Enfermagem UFPE on-line, 12(1), 102-11.

Beck, A. H. U. (2017). Psico-Oncologia: a atuação do psicólogo no tratamento de pacientes com câncer. Monografia. Universidade Regional do Noroeste do Estado do Rio Grande do Sul. , Ijuí - RS. Recuperado de http://bibliodigital. unijui.edu.br:8080/xmlui/handle/123456789/4849

Botega, N. J., Bio, M. R., Zomignami, M. A., Garcia., Jr., C., \& Pereira, W. A. B. (1995). Transtornos do humor em enfermaria de clínica médica e validação de escala de medida (HAD) de ansiedade e depressão. Revista de Saúde Pública. Recuperado de https://www.scielosp.org/article/rsp/1995. v29n5/359-363/

Boyle, C. C., Stanton, A. L., Ganz, A. P., \& Bower, J. E. (2017). Posttraumatic growth in breast cancer survivors: does age matter? Psycho-Oncology, 26: 800-807 Retrieved from https://onlinelibrary.wiley.com/doi/full/10.1002/ pon.4091 doi: 10.1002/pon.4091

Burguess, C., Cornelius, V., Love, S., Graham, J. Richards, M. \& Ramirez, A. (2005). Depression and anxiety in women with early breast cancer: five-year observational cohort study. The BMJ, Retrieved from https://www.bmj.com/ content/330/7493/702 doi: 10.1136/bmj.38343.670868.D3

Cardoso, L. L., Alberton, N. V. B., Fernandes, F. S., \& Castro, A. (2019). Percepção de familiares, crianças e adolescentes em Tratamento Oncológico, sobre o papel do Psicólogo. Revista multidisciplinar e de psicologia. 13 (46), 508-523. DOI: $10.14295 /$ id on-line. v13i46.1902

Casellas $\square$ Grau, A., Ochoa, C., \& Ruini, C. (2017). Psychological and clinical correlates of posttraumatic growth in cancer. A systematic and critical review. Psycho $\square$ oncology, 26(12), 2007-2018. Retrieved from https://onlinelibrary.wiley.com/doi/pdf/10.1002/pon.4426 doi: 10.1002/pon.4426

Castro, E. K. K. D., Lawrenz, P., Romeiro, F., Lima, N. B. D., \& Haas, S. A. (2016). Percepção da Doença e Enfrentamento em Mulheres com Câncer de Mama. Psicologia: Teoria e Pesquisa, 32(3), 1-6. Recuperado de http:// www.scielo.br/pdf/ptp/v32n3/1806-3446-ptp-32-03-e32324.pdf doi: 10.1590/0102-3772e32324

Couto, R. N. (2017). Perdão e Crescimento Pós-Traumático no âmbito do divórcio: uma explicação pautada nos valores humanos. Mestrado em psicologia social, Universidade Federal da Paraíba, João Pessoa - PB, Brasil. Recuperado de https://repositorio.ufpb.br/jspui/handle/tede/9087

Chen, H. M, Chen, V. C. H., Hsiao, HP., Weng, YP., Hsu, YT., Wung, JC., Su, JA., \& Chen, YL. (2019). Correlations and Correlates of Post-Traumatic Growth and Post-Traumatic Stress Symptoms. In Patients With Breast Cancer. Neuropsychiatric Disease and Treatment, 3051-3060. Retrieved from https://www.ncbi.nlm.nih.gov/pmc/articles/PMC6830357/ doi: 10.2147/ NDT.S218450

Choumanova, I., Wanat, S., Barret, R., \& Koopman. (2006). Religion and Spirituality in Coping with Breast Cancer: Perspectives of Chilean Women. The Breast Journal, 12(4), 349-352 Retrieved from https://onlinelibrary.wiley. com/doi/epdf/10.1111/j.1075-122X.2006.00274.x doi: 10.1111/j.1075122X.2006.00274.x

Dell'Aglio, D. D. (2000). O processo de coping, institucionalização e eventos de vida em crianças e adolescentes. Tese de doutorado. Universidade Federal do Rio Grande do Sul. Recuperado de http://pepsic.bvsalud.org/scielo. php?script $=$ sci_arttext\&pid $=$ S1413-389X2003000100005
Diab, S. Y., Isosävi, S., Qouta, S. R., Kuittinen, S., \&Punamäki, R. L. (2018). The protective role of maternal post-traumatic growth and cognitive trauma processing in Palestinian mothers and infants: a longitudinal study. The Lancet. Volume 39(2), S1-S53. Retrieved from https://www.thelancet.com/ journals/lancet/article/PIIS0140-6736(18)30405-7/fulltext doi: 10.1016/ S0140-6736(18)30405-7

Fonseca, C. C. (2011). Crescimento pós-traumático: o impacto de falar sobre os aspectos positivos de uma experiência traumática. Mestrado Integrado em Psicologia, Universidade de Lisboa. Recuperado de https://repositorio. ul.pt/bitstream/10451/4937/1/ulfpie039645_tm.pdf

Folkman, S. Lazarus, R. Gruen, R. J., \& DeLongis, A. (1986). Appraisal, coping, health status, and psychological symptoms. J. of Person. and Social Psychol., 50 (3), 571 - 579. Retrieved from https://www.ncbi.nlm.nih.gov/ pubmed/3701593 doi: 10.1037//0022-3514.50.3.571

Gandini, R. C., Martins, M. C. F., \& Pedrosa, E. C. M. (2008). Psicologia em estudo. Mini-Mac - escala de ajustamento mental para o câncer: estrutura fatorial 13(1), 169-177. Recuperado de http://www.scielo.br/pdf/pe/ v13n1/v13n1a19.pdf

Griep, R. H., Chor, D., Faerstein, E., Werneck, L., \& Lopes, C. S. (2005). Validade de constructo de escala de apoio social do Medical Outcomes Study adaptada para o português no Estudo Pró-Saúde. Cadernos de Saúde Pública. 21(3), 703-714. Recuperado de http://www.scielo.br/pdf/csp/ v21n3/04.pdf

Groarke, A., Curtis, R., Groarke, J. M., Hogan, M. J., Gibbons, A., \& Kerin, M. (2017). Post $\square$ traumatic growth in breast cancer: how and when do distress and stress contribute? Psycoonlogy, 26(7), 967-974. Retrieved from https:// onlinelibrary.wiley.com/doi/epdf/10.1002/pon.4243

Ho, S. M. Y., Chan, Y. W. M., Yau, T. K., \& Yeung, R. M. W. (2010). Relationships between explanatory style, posttraumatic growth and posttraumatic stress disorder symptoms among Chinese breast cancer patients. Psychology \& Health, 26 (3), 269-285. Retrieved from https://www.tandfonline.com/ doi/full/10.1080/08870440903287926 doi: 10.1080/0870440903287926

Hopman, P., \& Rijken, M. (2015). Illness perceptions of cancer patients: relationships with illness characteristics and coping. Psycho $\square$ Oncology, 24(1), 11-18. Retrieved from https://www.ncbi.nlm.nih.gov/pubmed/24891136 doi: $10.1002 /$ pon.3591

INCA -Instituto Nacional do Câncer. (2020). Estimativa 2020. Incidência de Câncer no Brasil. Recuperado de https://www.inca.gov.br/sites/ufu.sti. inca.local/files//media/document//estimativa-2020-incidencia-de-cancer-no-brasil.pdf

INCA - Instituto Nacional de Câncer. (2019). Câncer de mama: vamos falar sobre isso? (5 ed.). rev. atual. Rio de Janeiro. Recuperado de https://www. inca.gov.br/sites/ufu.sti.inca.local/files//media/document// cartilha-cancer-de-mama-vamos-falar-sobre-isso2016.pdf

Kroenke, C. H., Rosner, B., Chen, W. Y., Kawachi, I., Colditz, G.A., \& Holmes, M.D. (2004). Functional Impact of Breast Cancer by Age at Diagnosis. Journal of Clinical Oncology, 22(10), 1849-1856. Retrieved from https:// www.ncbi.nlm.nih.gov/pubmed/15143077 doi: 10.1200/JCO.2004.04.173

LaRocca, M. A., Scogin, F. R., Hilgeman, M. M., Smith, A. J., \& Chaplin, W. F. (2018). The impact of posttraumatic growth, transformational leadership, and self-efficacy on PTSD and depression symptom severity among combat Veterans. Military Psychology, 30(2), 162-173. Retrieved from https:// www.tandfonline.com/doi/full/10.1080/08995605.2018.1425073 doi: 10.1080/08995605.2018.1425073

Larsen, P.D \& Hummel, F.I. (2008). Adaptation. In: Larsen, PD \& Lubkin, IM. Chronic Illness: Impact and Intervention. $7^{\mathrm{a}} \mathrm{Ed}$. Sudbury: Jones \& Bartlett Learning.

Lawrenz, P., Castro, E. K. (2011). Enfrentamento religioso/espiritual diante do diagnóstico e tratamento do câncer de mama: um estudo piloto. Faculdade de Psicologia, universidade do Vale do Rio dos Sinos - UNISINOS.

Medeiros, E. D., Couto, R. N., Fonseca, P. N., Brito, R. C. S., \& Castro, L. S. (2016). Correlatos valorativos do crescimento pós-traumático em uma amostra brasileira. Psicologia e Saber Social, 5(2), 112-125. Recuperado de https://www.e-publicacoes.uerj.br/index.php/psi-sabersocial/article/ view/21602 doi: 10.12957/psi.saber.soc.2016.21602

Ministério da Saúde (1998) Portaria n. 3.535, de 2 de setembro de 1998. Estabelece critérios para cadastramento de centros de atendimento em oncologia. Recuperado de http://bvsms.saude.gov.br/bvs/saudelegis/ gm/1998/prt3535_02_09_1998_revog.html 
Hasson-Ohayon-, I., Tuval-Mashiach, R., Goldzweig, G., \& Levi, R. Pizem, N \& Kaufman, B. (2016). The need for friendships and information: Dimensions of social support and posttraumatic growth among women with breast cancer. Palliative \& Supportive Care. 14(4), 387-392. https:// doi.org/10.1017/S1478951515001042

Prati, G., \& Pietrantoni, L. (2009). Optimism, social support, and coping strategies as factors contributing to posttraumatic growth: A Meta-Analysis. Journal of loss and trauma, 14(5), 364-388. Retrieved from https://www.tandfonline.com/doi/full/10.1080/15325020902724271 doi: $10.1080 / 15325020902724271$

Raz-Hamama, Y., Pat-Horenczyk, R., Roziner, I., Perry, S., \& Stemmer, M. S. (2019). Can posttraumatic growth after breast cancer promote positive coping? -A cross-lagged study. Psycooncology, 28(4), 767-774. Retrieved from https://pubmed.ncbi.nlm.nih.gov/30720241/ doi: 10.1002/pon.5017

Reich, M., Gaudron, C., Penel, N. (2009). When cancerophobia and denial lead to death. Palliative and Supportive Care; 7(2), 253-255. Retrieved from https://www.cambridge.org/core/journals/palliative-and-supportive-care/article/when-cancerophobia-and-denial-lead-to-death/FE5C15C8615940D832A45F5F3F00F015 doi: 10.1017/S1478951509000327

Rossi, L., \& Santos, M. A. (2003). Repercussões Psicológicas do Adoecimento e Tratamento em Mulheres Acometidas pelo Câncer de Mama. Psicologia ciência e profissão, 23(4), 32-41. Recuperado de http://www.scielo.br/scielo. php?script $=$ sci_abstract\&pid $=$ S1414-98932003000400006\&lng $=$ en\&nrm $=$ iso\&tlng=pt doi: $10.1590 /$ S1414-98932003000400006.

Salsman, J. M., Segerstrom, S. C., Brechting, E. H., Carlson, C. R., \& Andrykowski, M. A. (2008). Posttraumatic growth and PTSD symptomatology among colorectal cancer survivors: a $3 \square$ month longitudinal examination of cognitive processing. Psycho-Oncology, Retrieved from https://onlinelibrary. wiley.com/doi/abs/10.1002/pon.1367 doi: 10.1002/pon.1367

Santos., Jr., R., Simone, C. S., Pinhabel, G. V., Galhardo, V. A. T., \& Carniel, A., 2019. Sentido de vida e saúde mental durante o tratamento de câncer. Mudanças - Psicologia da Saúde, 26 (2), 17-24. Recuperado de https://www. metodista.br/revistas/revistas-ims/index.php/MUD/article/view/8650 doi: 10.15603/2174-1019

Santos, M. (2017). Trauma Psicológico e resiliência: relação com o tipo de evento potencialmente traumático e o crescimento pós-traumático. Mestrado Integrado em Psicologia, Universidade de Lisboa. Recuperado de https:// repositorio.ul.pt/bitstream/10451/33203/1/ulfpie052852_tm.pdf

Sartore, A. C., \& Grossi, S. A. A. (2008). Escala de Esperança de Herth - instrumento adaptado e validado para a língua portuguesa. Revista da Escola de Enfermagem da USP. Recuperado de http://www.scielo.br/scielo. php?script=sci_arttext\&pid=S0080-62342008000200003\#nt

Scannavino, C. S. S., Sorato, D. B., Lima, M. P., Franco, A. H. J., Martins, M. P., Morais., Jr, J. C., Bueno, P. R. T., Rezende, F, F., \& Valério, N. I. (2013). Psico-Oncologia: atuação do psicólogo no Hospital de Câncer de Barretos. Psicologia USP, São Paulo, 2013, 24(1), 35-53 Recuperado de http://www. scielo.br/scielo.php?script=sci_arttext\&pid $=$ S0103-65642013000100003 doi: 10.1590/S0103-65642013000100003

Schroevers, MJ., Helgeson, V. S., Sanderman, R., \& Ranchor, A. V. (2010). Type of social support matters for prediction of posttraumatic growth among cancer survivors. Psycho $\square$ Oncology, 19(1), 46-53. Retrieved from https://www. ncbi.nlm.nih.gov/pubmed/19253269 doi: 10.1002/pon.1501.

Silva, A. V., Zandonade E., \& Amorim M. H. C. (2017). Ansiedade e o enfrentamento de mulheres com câncer de mama em quimioterapia Rev. Latino-Am. Enfermagem, 25. Retirado de http://www.scielo. br/scielo.php?script=sci_arttext\&pid=S0104-11692017000100343\&lng=e n\&tlng=en doi: $10.1590 / 1518-8345.1722 .2891$

Silva, D. (2019). Considerações e reflexões sobre o psicodiagnóstico de stress em pacientes com câncer. Faculdade Sant'Ana em Revista, 5 (1), 21-35. Recuperado de https://www.iessa.edu.br/revista/index.php/fsr/article/view/1235

Silva, P. A \& Melo-Santos, C. (2008). Reação de ajustamento em oncologia. In: Carvalho, VA et al. Temas em psico-oncologia. São Paulo: Summus.
Tavares, J.S.C.; Trad, L.A.B. (2009) Families of women with breast cancer: challenges associated with care and coping resources. Interface - Comunic, Saúde, Educ.,.13(29), 395-408. Recuperado de http://www.scielo.br/pdf/ icse/v13n29/v13n29a12.pdf

Tedeschi, R. G. \& Calhoun, L. G. (1996). The posttraumatic growth inventory: Measuring the positive legacy of trauma. Journal of traumatic Stress. 9(3), 455-471. Retrieved from https://onlinelibrary.wiley.com/doi/abs/10.1002/ jts.2490090305 doi: 10.1002/jts.2490090305

Tedeschi, R. G. \& Calhoun, L. G. (2004). TARGET ARTICLE: "Posttraumatic Growth: Conceptual Foundations and Empirical Evidence", Psychological Inquiry, 15(1), 1-18. Retrieved from https://drive.google.com/ drive/folders/1kUs0h0aUPanhphIFN-8Iu3qqfYQmFT1D doi: 10.1207/ s15327965pli1501_01

Tobin, J., Allem, J. P., Slaughter, R., Unger, J. B., Hamilton, A. S., Millam, J. E. (2017). Posttraumatic growth among childhood cancer survivors: Associations with ethnicity, acculturation, and religious service attendance. Journal of Psychosocial Oncology , 36(2), 175-188. Retrieved from https:// www.tandfonline.com/doi/full/10.1080/07347332.2017.1365799 doi: 10.1080/07347332.2017.1365799

Todorov, N., Sherman, K. A., \& Kilby, C. J. (2019). Self compassion and hope in the context of body image disturbance and distress in breast cancer survivors. Psycho-Oncology, 2025-2032. Retrieved from https://researchers. mq.edu.au/en/publications/self-compassion-and-hope-in-the-context-of-body-image-disturbance

Val, B. E., \& Linley, P. A., (2006). Posttraumatic Growth, Positive Changes, and Negative Changes in Madrid Residents following the March 11, 2004, Madrid Train Bombings. Journal of Loss and Trauma, 11(5), 409-424. Retrieved from https://www.tandfonline.com/doi/ full/10.1080/15325020600685519

Veras, L. A. T., Veras, Jr., \& Carvalho, P. M. G. D. C. (2015). Enfrentamento e resiliência de pacientes com câncer submetidos a tratamento quimioterápico. Revista Interdisciplinar, 8(2), 195-201. Recuperado de https://revistainterdisciplinar.uninovafapi.edu.br/index.php/revinter/article/view/574

Wang, M. L., Liu, JE., Wang, H. Y., Chen, J., \& Li, Y. Y. (2014). Posttraumatic growth and associated socio-demographic and clinical factors in Chinese breast cancer survivors. European Journal of Oncology Nursing, 18(5), 478483. Retrieved from https://www.ncbi.nlm.nih.gov/pubmed/24958639 doi: 10.1016/j.ejon

Zanini, D. S., Primi, T. C. N., \& Peixoto, E. (2018). Escala de Apoio Social (MOS-SSS): Proposta de Normatização com Referência nos Itens. Teorias em Psicologia, 26(1), 387-399 Recuperado de https://www.researchgate.net/ publication/324134701_Escala_de_Apoio_Social_MOS-SSS_Proposta_de_ Normatizacao_com_Referencia_nos_Itens doi 10.9788/TP2018.1-15Pt

Zeligman, M., Varney, M., Grad, R. I., \& Huffstead, M. (2018). Posttraumatic Growth in Individuals with Chronic Illness: The Role of Social Support and Meaning Making. Journal of Counseling \& Development, 96(1), 53-63. Retrieved from https://onlinelibrary.wiley.com/doi/full/10.1002/jcad.12177 doi: $10.1002 /$ jcad.12177

Zhou, X., Wu, X., \& Zhen, R. (2018). Self-esteem and hope mediate the relations between social support and post-traumatic stress disorder and growth in adolescents following the Ya'an earthquake. Anxiety, Stress, \& Coping, 31(1), 32-45. Retrieved from https://www.tandfonline.com/doi/full/10.1 080/10615806.2017.1374376 doi: 10.1080/10615806.

Zigmond, A. S. \& Snaith, R. P. (1983). The hospital anxiety and depression scale. Acta Psychiatrica Scandinavica, 67(6), 361-370. Retrieved from https:// www.ncbi.nlm.nih.gov/pubmed/6880820 doi 10.1111/j.1600-0447.1983. tb09716.x

Submetido em: 26-2-2020

Aceito em: 20-9-2020 